\title{
Hepatotoxicity of immune checkpoint inhibitors: a histology study of seven cases in comparison with autoimmune hepatitis and idiosyncratic drug-induced liver injury
}

\author{
Yoh Zen ${ }^{1} \cdot$ Matthew M. Yeh $\mathbb{D}^{2}$
}

Received: 29 September 2017 / Revised: 12 November 2017 / Accepted: 3 December 2017 / Published online: 5 February 2018

(c) United States \& Canadian Academy of Pathology 2018

\begin{abstract}
The adverse effects of immune checkpoint inhibitors in various organs may be attributed to immune-mediated processes triggered by disrupted self-tolerance; however, it remains unclear whether they are similar or dissimilar to classic organspecific autoimmune diseases. The present study aimed to compare clinicopathologic features between checkpoint inhibitorinduced liver injury and acutely presenting autoimmune hepatitis or idiosyncratic drug-induced liver injury. Seven patients treated with nivolumab $(n=5)$ or ipilimumab $(n=2)$ presented with liver dysfunction a median of 41 days (range 21-120) after the initiation of immunotherapy. All patients had elevated liver enzymes, whereas hyper-bilirubinemia was less common. None of the patients had antinuclear antibodies or IgG elevations. Stopping the immunotherapy and additional immunosuppression with corticosteroids normalized or decreased liver enzymes in all patients treated. Histologically, all biopsies showed predominantly lobular hepatitis with milder portal inflammation. Centrilobular confluent necrosis and plasmacytosis were observed in a single case, and were markedly less common and milder than those in autoimmune hepatitis $(p=0.017$ and $p<0.001$, respectively). Bile duct injury, micro-abscesses, and extramedullary hematopoiesis were also found in one case each. Immunostaining revealed the presence of large numbers of CD3+ and CD8+ lymphocytes, whereas CD20 + B cells and CD4+ T cells were fewer in checkpoint inhibitor-induced liver injury than in autoimmune hepatitis or drug-induced liver injury. In conclusion, liver injury caused by cancer immunotherapy shares some features with injury of autoimmune hepatitis; however, there are obvious differences between the two conditions. Checkpoint inhibitorinduced liver injury may represent an immune-mediated, less zone-selective hepatocyte necrosis not requiring the strong activation of helper $\mathrm{T}$ cells and immunoglobulin production.
\end{abstract}

\section{Introduction}

Immunomodulation using immune checkpoint inhibitors has become a promising approach for the treatment of various malignant neoplasms $[1,2]$. This emerging immunotherapy was originally used for advanced malignant melanoma, but has since been approved or is currently being tested in clinical trials for many other malignancies including nonsmall-cell lung cancers and renal cell carcinomas [3-8].

Yoh Zen

yohzen@med.kobe-u.ac.jp

1 Department of Diagnostic Pathology, Kobe University Graduate School of Medicine, Kobe, Japan

2 Department of Pathology, University of Washington School of Medicine, Seattle, WA, USA
Nivolumab and ipilimumab are now the two most commonly used checkpoint inhibitors [2, 9, 10]. Nivolumab recognizes programmed cell death 1 (PD-1), mainly expressed by $\mathrm{T}$ lymphocytes, and blocks the interaction PD1 and its ligand, programmed death ligand 1 (PD-L1) expressed on cancer cells [1, 11]. The blockage of this ligand-receptor interaction inhibits the inactivation of $\mathrm{T}$ lymphocytes and enhances anticancer cytotoxic effects. Ipilimumab targets cytotoxic T-lymphocyte-associated antigen 4 (CTLA4) on the surface of T lymphocytes [11]. Since the CTLA4 signal also inactivates lymphocytes, blocking CTLA4 by ipilimumab enables $\mathrm{T}$ lymphocytes to exert their cytotoxic effects on tumor cells [12]. Although PD-1 and CTLA4 are mainly expressed by CD8+ cytotoxic T cells, other lymphocytes including regulatory $\mathrm{T}$ cells and $\mathrm{CD} 4+$ $\mathrm{T}$ cells are also suggested to have these receptors [13-15].

Checkpoint inhibitors sometimes induce various adverse effects including skin rashes, colitis, pancreatitis, nephritis, 
and hepatitis [16, 17]. Based on the assumption that T-cell inactivation by the PD-1 and CTLA4 pathways is also involved in immune tolerance to self-antigens, adverse effects may be triggered by autoimmune reactions. Therefore, the side effects of checkpoint inhibitors are often referred to as "immune-mediated adverse events" in order to distinguish them from usual idiosyncratic drug-induced organ damage [16]. Although liver injuries caused by nivolumab and ipilimumab were originally suspected to resemble classic autoimmune hepatitis [18], recent pathology papers raised the possibility that clinicopathologic features of immunotherapy-related hepatitis may not be identical to those of autoimmune hepatitis [19, 20]. However, these two conditions have not yet been systematically compared.

In the present study, the biopsy findings of liver injuries caused by nivolumab or ipilimumab were compared with classic autoimmune hepatitis and idiosyncratic druginduced liver injury in order to elucidate how checkpoint inhibitor-induced liver injuries are similar or dissimilar to the two other conditions.

\section{Materials and methods}

\section{Patients}

This study received ethical approval from Kobe University Graduate School of Medicine. A search of the pathology database at our institutes and consultation files of the authors identified seven cases of liver injuries caused by nivolumab $(n=5)$ and ipilimumab $(n=2)$. Five were Japanese cases, while the remaining two were selected from the database in Seattle. Electronically stored clinical records and histology slides were retrospectively reviewed.

Ten cases each of autoimmune hepatitis and drug-induced liver injury were also selected for comparison (Table 1). Consecutive cases of autoimmune hepatitis that clinically showed an acute presentation were chosen because liver injuries due to checkpoint inhibitors were sudden-onset hepatitis. All selected patients with autoimmune hepatitis had $>1: 40$ titers of antinuclear antibodies and IgG elevations with clinicopathologic features meeting the criteria of definite autoimmune hepatitis based on the simplified scoring system [21]. As for drug-induced liver injury, consecutive cases of predominantly hepatitic injury were selected. All cases of drug-induced liver injury were clinically determined to be idiosyncratic and not due to drug overdose. Causative drugs were antibiotics $(n=3)$, anti-inflammatory agents $(n$ $=3$ ), anti-convulsants $(n=2)$, and herbal medicine $(n=2)$. All patients with autoimmune hepatitis or drug-induced liver injury were negative for viral markers, with cases of druginduced liver injury also lacking antinuclear antibodies.

\section{Evaluation of liver biopsies}

Liver biopsies were reviewed in terms of the following findings: portal inflammation, lobular injury, confluent necrosis, eosinophilic infiltration, and plasmacytosis. The degrees of portal inflammation, lobular injury, and confluent necrosis were examined according to Ishak's scoring system for chronic hepatitis [22]. Although interface hepatitis and fibrosis were also a part of Ishak's scoring scheme, they are rare in acute-onset liver diseases; therefore, these findings were not examined in the present study. Although Ishak's scoring system is designed for chronic hepatitis, it was selected in this study because this scheme allows to evaluate multiple hepatitic findings in a semiquantitative manner and also because of the lack of reliable scoring systems for acute hepatitis. The presence or absence of $>5$ eosinophils per high power field and $>10$ plasma cells per high power field was evaluated. Other features such as bile duct injury were also recorded if present.

\section{Immunohistochemistry}

All biopsy specimens were fixed in $10 \%$ buffered formalin and embedded in paraffin. Unstained slides for immunohistochemistry were available in five nivolumab-treated

Table 1 Characteristics of patients examined in this study

\begin{tabular}{lllll}
\hline Conditions & $\begin{array}{l}n \\
\text { (range) }\end{array}$ & $\begin{array}{l}\text { Gender (Male/ } \\
\text { female) }\end{array}$ & Details \\
\hline $\begin{array}{l}\text { Checkpoint inhibitor-associated liver } \\
\text { injury }\end{array}$ & 7 & $61(24-76)$ & $5 / 2$ & $\begin{array}{l}\text { Liver injuries caused by nivolumab }(n=5) \text { and } \\
\text { ipilimumab }(n=2) \text {. A clinical summary is available in } \\
\text { Table } 2\end{array}$ \\
Autoimmune hepatitis & $1066(20-80)$ & $2 / 8$ & $\begin{array}{l}\text { All showed an acute presentation, and had antinuclear } \\
\text { antibodies and IgG elevations, meeting the criteria for } \\
\text { definite autoimmune hepatitis } \\
\text { All presented with predominantly hepatitic liver } \\
\text { dysfunction. Causative drugs were antibiotics }(n=3), \\
\text { anti-inflammatory agents }(n=3), \text { anti-convulsants } \\
(n=2), \text { and herbal medicine }(n=2)\end{array}$ \\
\hline
\end{tabular}


cases and all cases of autoimmune hepatitis or druginduced liver injury. Immunostaining was performed on a Ventana Benchmark XT (Ventana Medical Systems, Inc., Tucson, AZ) or Bond Max autostainer (Leica Microsystems, Wetzlar, Germany) according to the manufacturers' protocols. Deparaffinized sections were heattreated and incubated with primary antibodies. Immunohistochemical staining for multiple lymphocyte markers was performed using the following antibodies: CD3 (clone F7.2.38, 1:50, Dako Cytomation, Glostrup, Denmark), CD4 (clone 1F6, prediluted, Leica Microsystems), CD8 (clone SP57, Roche, Ltd., Basel, Switzerland), and CD20 (clone L26, prediluted, Dako Cytomation). The numbers of lymphocytes positive for each marker were counted at three hot spots and calculated as the average per high power field. Ratios of CD20+/CD3+ and CD4 $+/ \mathrm{CD} 8+$ cells were also calculated in each case.

\section{Statistical analysis}

Statistical analyses were performed using the $\chi^{2}$, Fisher's, or Mann-Whitney $U$-test. A $p$ value $<0.05$ was considered to be significant. Statistical analyses were performed using SPSS software (IBM, Chicago, IL).

\section{Results}

\section{Clinical features}

Clinical features are summarized in Table 2. The study cohort consisted of five men and two women with a median age of 61 years (range 24-76). Five and two patients received nivolumab and ipilimumab monotherapy, respectively, for the management of advanced malignant melanomas $(n=6)$ or squamous cell carcinoma of the tongue ( $n$ $=1$ ). Patient 2 had a history of primary biliary cholangitis, which had been treated with ursodeoxycholic acid for 13 years. Patient 5 was diagnosed as having rheumatoid arthritis 3 years before, and was treated with methotrexate. He was symptom-free without medication when immunotherapy for lingual cancer started.

Liver dysfunction was observed after the administration of 1 to 6 doses of checkpoint inhibitors. Durations between the initiation of immunotherapy and onset of liver dysfunction ranged between 21 and 120 days (median 41). All patients presented with elevated liver enzymes. The severities and patterns of serological abnormalities varied widely. Hyper-bilirubinemia was less common than elevated liver enzymes (Table 2). All patients were negative for antinuclear antibodies, and elevated IgG was also not observed in any of the six cases tested. None had manifestations of checkpoint inhibitor-related injury in other

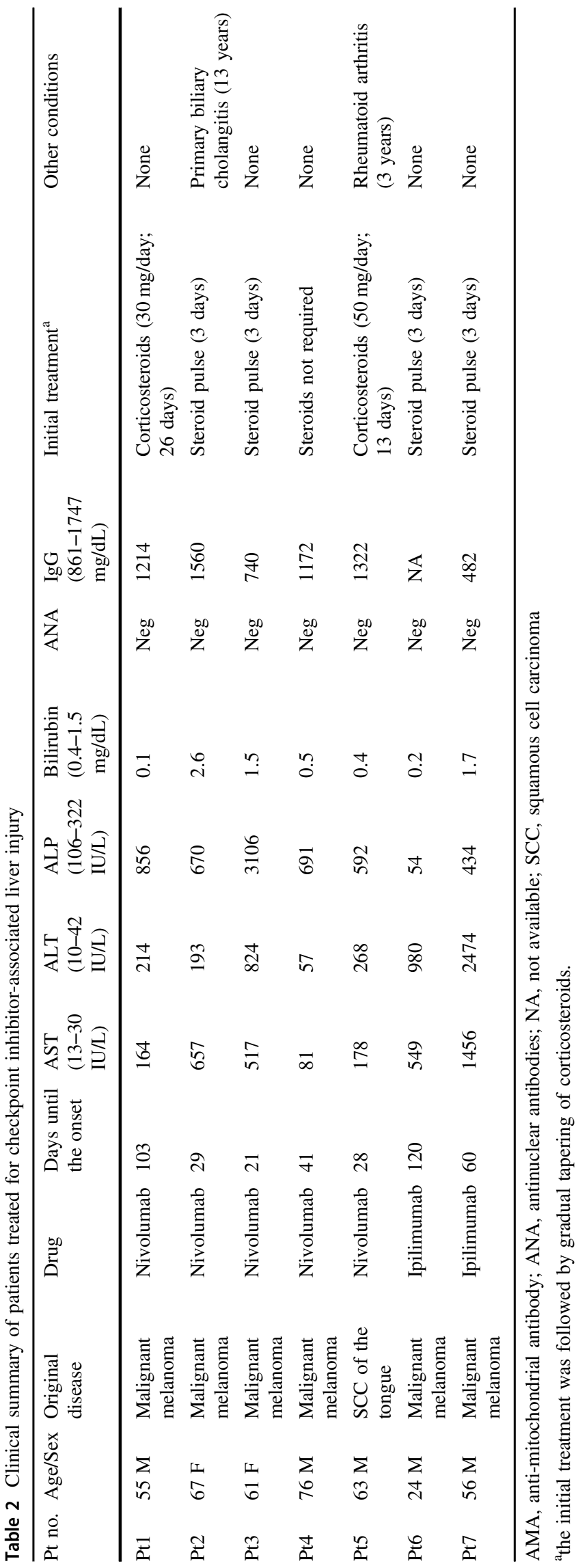


organs. Liver injuries required stopping the immunotherapy and the initiation of corticosteroid therapy in all, but patient 4 , for whom serological abnormalities improved by only stopping the administration of nivolumab. Four patients received steroid pulse (methylprednisolone $1,000 \mathrm{mg} / \mathrm{day}$, 3 days), while patients 1 and 5 had prednisolone (30 and 50 $\mathrm{mg} /$ day) for 26 and 13 days, respectively (Table 2). Although patient 7 continued to have mild serological liver dysfunction after steroid pulse therapy, all other treated patients showed good responses to the initial steroid therapy. After serological improvement of liver dysfunction was confirmed, corticosteroids were gradually tapered off in the following 2 weeks to 7 months.

\section{Liver biopsy findings}

The microscopic findings of liver biopsies are summarized in Table 3. All cases predominantly exhibited lobular hepatitis with milder portal inflammation. Except for patient 5 , lobular injury consisted of randomly scattered foci of focal necrosis and acidophilic bodies with no confluent necrosis (Fig. 1a, b). Patient 5 also had centrilobular zonal necrosis (Fig. 1c). Focal aggregates of eosinophils ( $>5$ cells/ high power field) were observed in two cases. Plasmacytic infiltration was not conspicuous in any cases. Patient 5 had $>10$ plasma cells per high power field, whereas the degree of plasmacytosis was less than that expected in classic autoimmune hepatitis. Both cases of ipilimumab-associated liver injury had foci of macrophage aggregation; however, this finding was not observed in nivolumab-treated cases. No discrete granuloma was found in any cases.

Patient 1 also exhibited changes suggestive of large-duct biliary injury, such as a ductular reaction, biliary-type interface activity, and cholangiolitis (Fig. 1d). He did not have any imaging features suggestive of metastatic melanoma involving the biliary tree. In patient 3 , many microabscesses were present in the parenchyma, but immunostaining for cytomegalovirus was negative (Fig. 1e). The liver biopsy sample of patient 4 showed extramedullary hematopoiesis, suggesting bone marrow injury (Fig. 1f). Further clinical evaluations with a bone marrow biopsy eventually identified bone marrow involvement in malignant melanoma. Patient 2 with known primary biliary cholangitis showed the features of chronic cholangiopathy such as bile duct injury, early ductopenia, and copperassociated protein deposition, the overall portal tract changes consistent with primary biliary cholangitis stage 3 .

\section{Comparison with autoimmune hepatitis and drug- induced liver injury}

All, but one case of autoimmune hepatitis showed predominantly lobular hepatitis, with eight cases also being

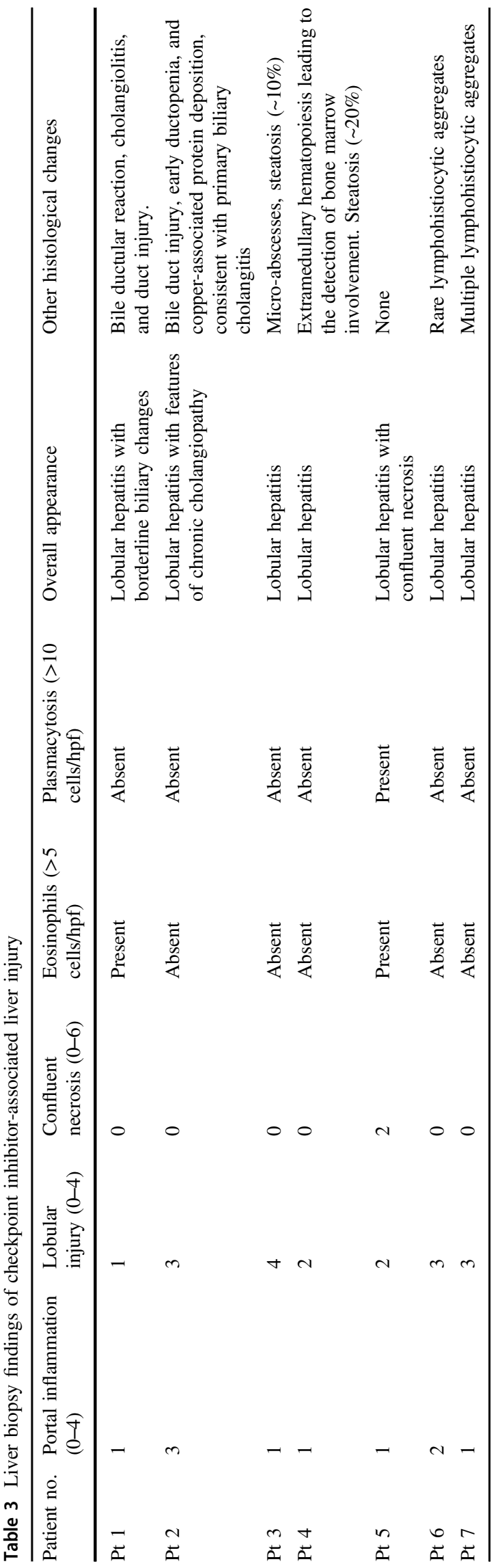


Fig. 1 Histologic features of checkpoint inhibitor-induced liver injury. The liver parenchyma is mainly damaged, with many foci of focal necrosis and acidophilic bodies being observed $(\mathbf{a}, \mathbf{b})$. One case each also showed centrilobular perivenular zonal cell loss (c, patient 5), ductular reaction with neutrophilic infiltration (d, patient 1), many foci of microabscesses (e, patient 3), and extramedullary hematopoiesis (f, patient 4$)$. The arrow and arrowheads in image $\mathbf{f}$ indicate a megakaryocyte and other hematopoietic cells, respectively
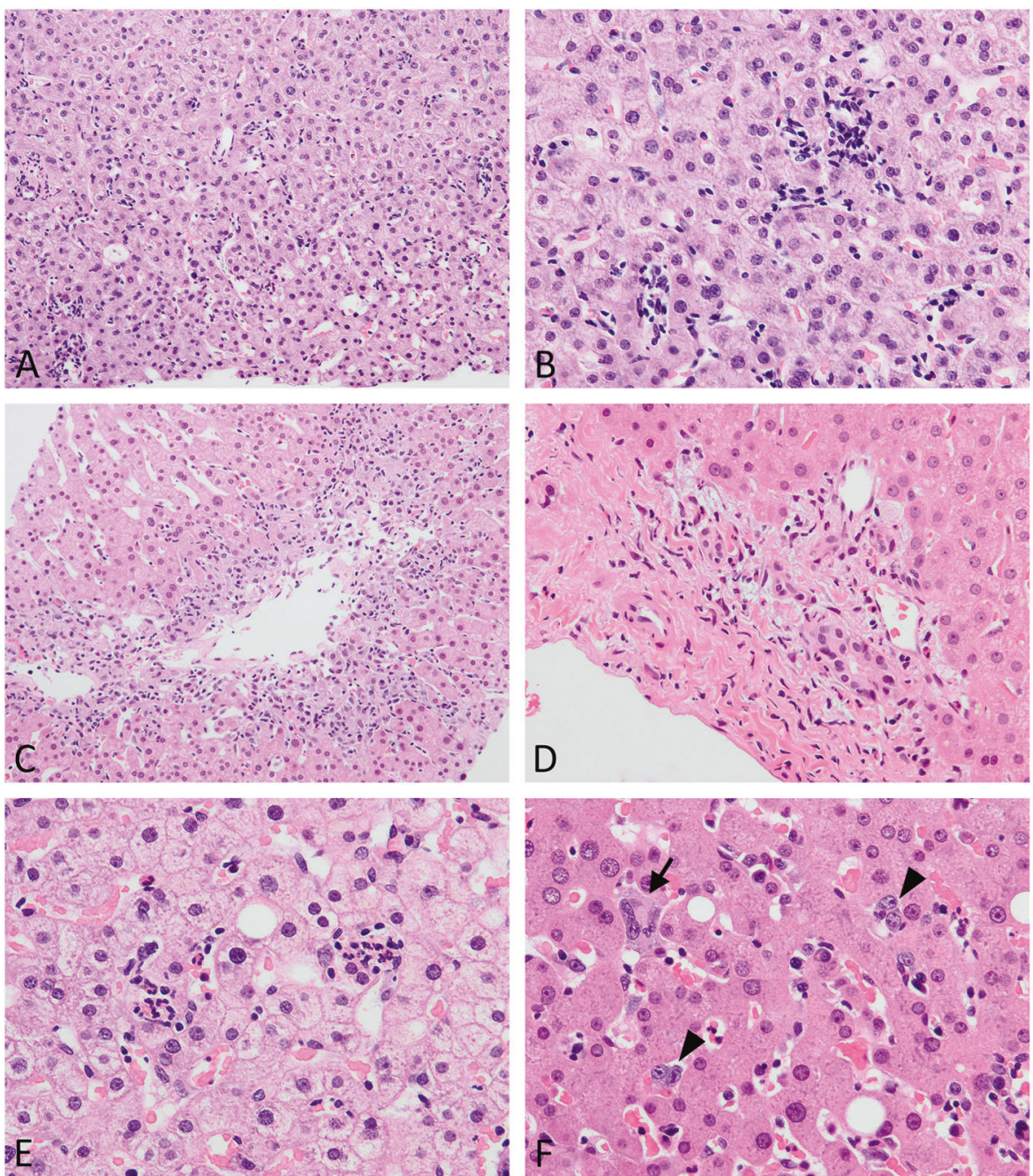

associated with confluent necrosis of variable degrees. Although the remaining case of autoimmune hepatitis clinically presented with acute illness, the histology of the liver biopsy sample was active lobular hepatitis with dense portal inflammation and interface hepatitis, suggesting the acute exacerbation of chronic hepatitis. All cases of druginduced liver injury also showed predominantly lobular inflammation with or without confluent necrosis.

The histologic features of immune checkpoint inhibitorassociated liver injury were compared with those of autoimmune hepatitis or drug-induced liver injury (Fig. 2). The degrees of portal inflammation and lobular injury did not significantly differ among the three disorders. Confluent necrosis in checkpoint inhibitor-associated liver injury was less common and milder than that in autoimmune hepatitis ( $p$ $=0.017)$ and drug-induced liver injury $(p=0.070)$. Eosinophilic infiltration was also less common in immunotherapytriggered hepatitis than in drug-induced liver injury $(p=$ 0.034). Plasmacytosis was always observed in autoimmune hepatitis, but in only $2 / 7$ (29\%) cases of checkpoint inhibitor- associated liver injury $(p<0.001)$. Bile plugs were slightly more common in drug-induced liver injury than in checkpoint inhibitor-induced liver injury $(p=0.056)$. Hepatocellular rosettes and emperipolesis were observed in 4/10 (40\%) and $8 / 10(80 \%)$ cases of autoimmune hepatitis, but none of immunotherapy-related liver injury $(p=0.056$ and 0.001 , respectively). Multinucleated hepatocytes were present in $8 /$ $10(80 \%)$ cases of autoimmune hepatitis and $3 / 7(43 \%)$ cases of checkpoint inhibitor-induced liver injury $(p=0.115)$. Small discrete granulomas were found in a case of druginduced liver injury but none of immunotherapy-related liver disease $(p=0.853)$.

\section{Characterization of infiltrating lymphocytes}

The results of immunohistochemistry are summarized in Fig. 3. Checkpoint inhibitor-associated liver injury was characterized by the infiltration of predominantly $\mathrm{CD} 3+$ and CD8+ lymphocytes, while fewer CD20+ or CD4+ lymphocytes were detected (Fig. 4). 


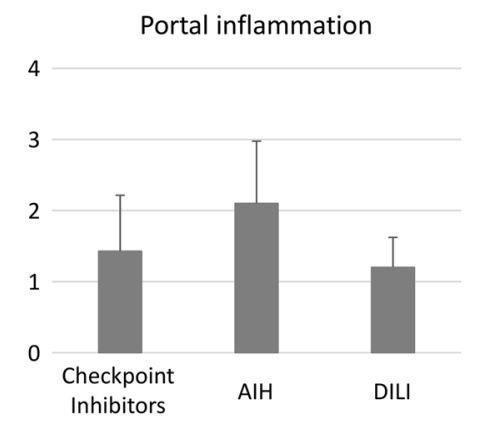

Eosinophilic infiltration

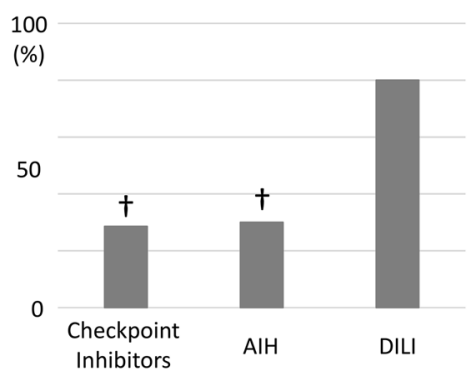

Fig. 2 Comparisons of histopathologic features among checkpoint inhibitor-induced liver injury, autoimmune hepatitis (AIH), and druginduced liver injury (DILI). Six histologic findings were scored in individual cases: portal inflammation, lobular injury, confluent necrosis, eosinophilic infiltration ( $>5$ cells/high power field),

A comparison between checkpoint inhibitor-induced liver injury and autoimmune hepatitis revealed that the number of $\mathrm{CD} 20+, \mathrm{CD} 3+, \mathrm{CD} 4+$, or CD8+ lymphocytes was smaller in checkpoint inhibitor-associated hepatitis than in autoimmune hepatitis. This trend was particularly obvious for CD20+ or CD4+ lymphocytes (both $p=0.003$ ). Therefore, $\mathrm{CD} 20+/ \mathrm{CD} 3+$ and $\mathrm{CD} 4+/ \mathrm{CD} 8+$ cell ratios in checkpoint inhibitor-induced liver injury were also markedly lower than those in autoimmune hepatitis $(p=0.008$ and $p=0.003$, respectively). In autoimmune hepatitis, CD20+ or CD4+ were predominantly present in enlarged portal tracts and periportal parenchyma (Fig. 5). Although all cases of autoimmune hepatitis showed an acute clinical presentation, small lymphoid aggregates containing CD20+ lymphocytes were observed in some portal tracts.

The difference between checkpoint inhibitor-associated liver injury and drug-induced liver injury was less conspicuous. However, the number of CD20+ and CD4+ lymphocytes in checkpoint inhibitor-related liver disease was even smaller than that in drug-induced liver injury ( $p=$ 0.017 and $p=0.003$, respectively).

\section{Discussion}

To the best of our knowledge, this is the first clinical and pathologic study systematically comparing checkpoint
Lobular injury

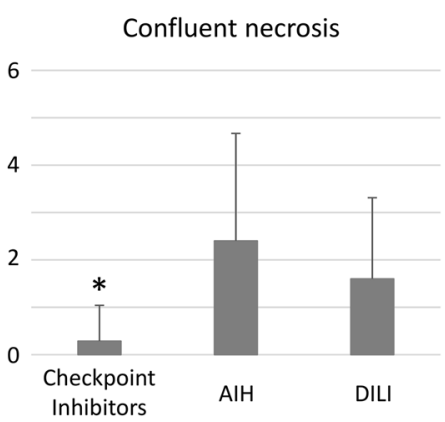

Bile plugs

100

(\%)

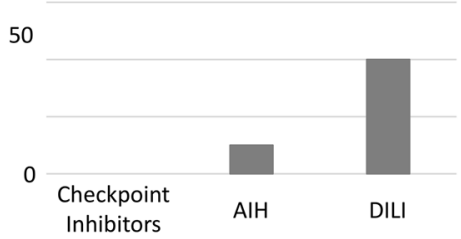

plasmacytosis ( $>10$ cells/high power field), and bile plugs. The former three findings are scored based on Ishak's system, while the latter three are shown as the $\%$ of cases having each microscopic finding. ${ }^{*} p<$ 0.05 vs. $\mathrm{AIH} ;{ }^{\dagger} p<0.05$ vs. DILI

inhibitor-induced liver injury with autoimmune hepatitis and drug-induced liver injury. In addition, although a few case reports described liver biopsy findings of nivolmabrelated liver damage [23], this is the first case series with indepth histopathological analyses. In contrast, three previous studies examined the histopathologic features of ipilimumab-related hepatitis [18-20]. Among the 18 reported cases of ipilimumab-related hepatitis with detailed histopathologic features available, 16 showed lobular hepatitis, with six also being associated with confluent necrosis [18-20]. The overall pattern of liver injuries appeared to be similar between ipilimumab- and nivolumabinduced hepatitis, whereas confluent necrosis may be less common in the latter. Seven reported cases of ipilimumabinduced hepatitis also had conspicuous histiocytes in sinusoids, with some forming granuloma-like aggregates [19, 20]. That finding was also observed in both ipilimumabtreated cases in our cohort, suggesting that histiocytes are activated more in ipilimumab-induced liver injury.

The present study identified similarities and dissimilarities between checkpoint inhibitor-induced liver injury and classic autoimmune hepatitis. Similar to most cases of acutely presenting autoimmune hepatitis, liver injury caused by checkpoint inhibitors exhibited lobular hepatitis with milder portal inflammation. Both conditions had many infiltrating T-lymphocytes positive for $\mathrm{CD} 3$ or $\mathrm{CD} 8$. Although low-grade liver dysfunction remained after steroid 
CD20

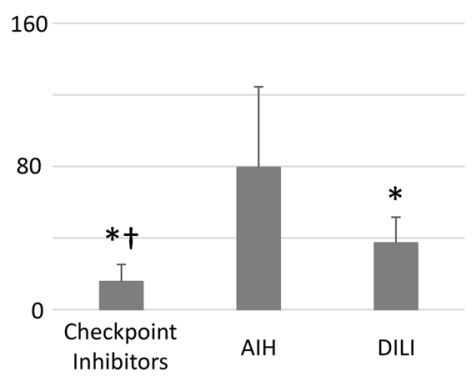

CD4

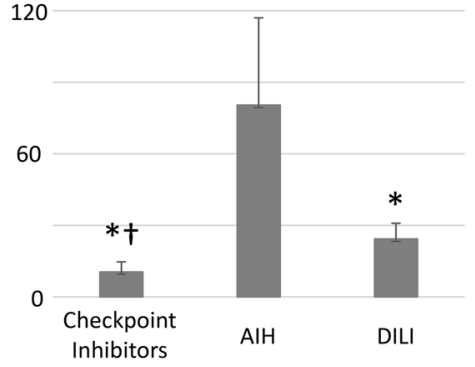

CD3

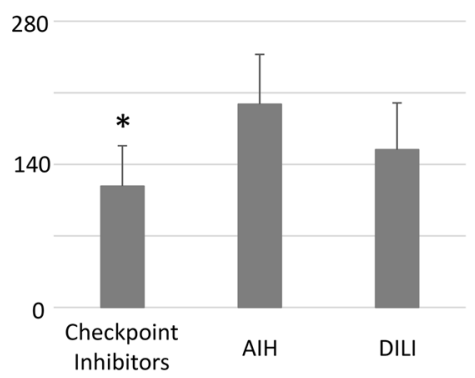

CD8

280

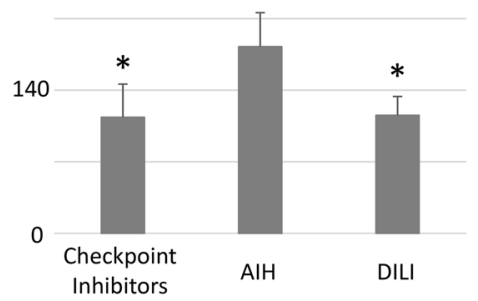

CD20/CD3

0.8

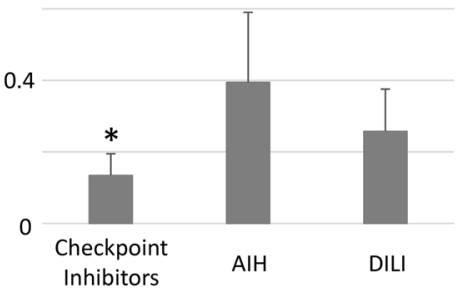

$\mathrm{CD} 4 / \mathrm{CD} 8$

0.8

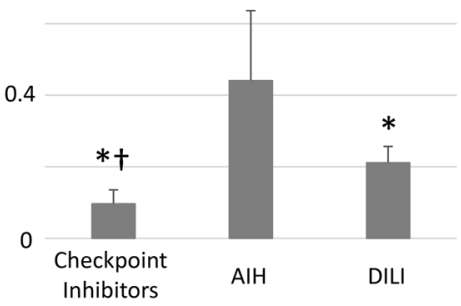

Fig. 3 Characterization of infiltrating inflammatory cells in checkpoint inhibitor-induced liver injury, autoimmune hepatitis (AIH), and druginduced liver injury (DILI). The absolute numbers of lymphocytes

positive for $\mathrm{CD} 20, \mathrm{CD} 3, \mathrm{CD} 4$, or $\mathrm{CD} 8$ were counted per high power field. The ratios of $\mathrm{CD} 20+/ \mathrm{CD} 3+$ or $\mathrm{CD} 4+/ \mathrm{CD} 8+$ cells were also calculated. $* p<0.05$ vs. AIH; ${ }^{\dagger} p<0.05$ vs. DILI

Fig. 4 Immunohistochemical results of checkpoint inhibitorinduced liver injury. The number of $\mathrm{CD} 20+$ or CD4+ lymphocytes was markedly smaller than that of CD3+ or CD8+ lymphocytes
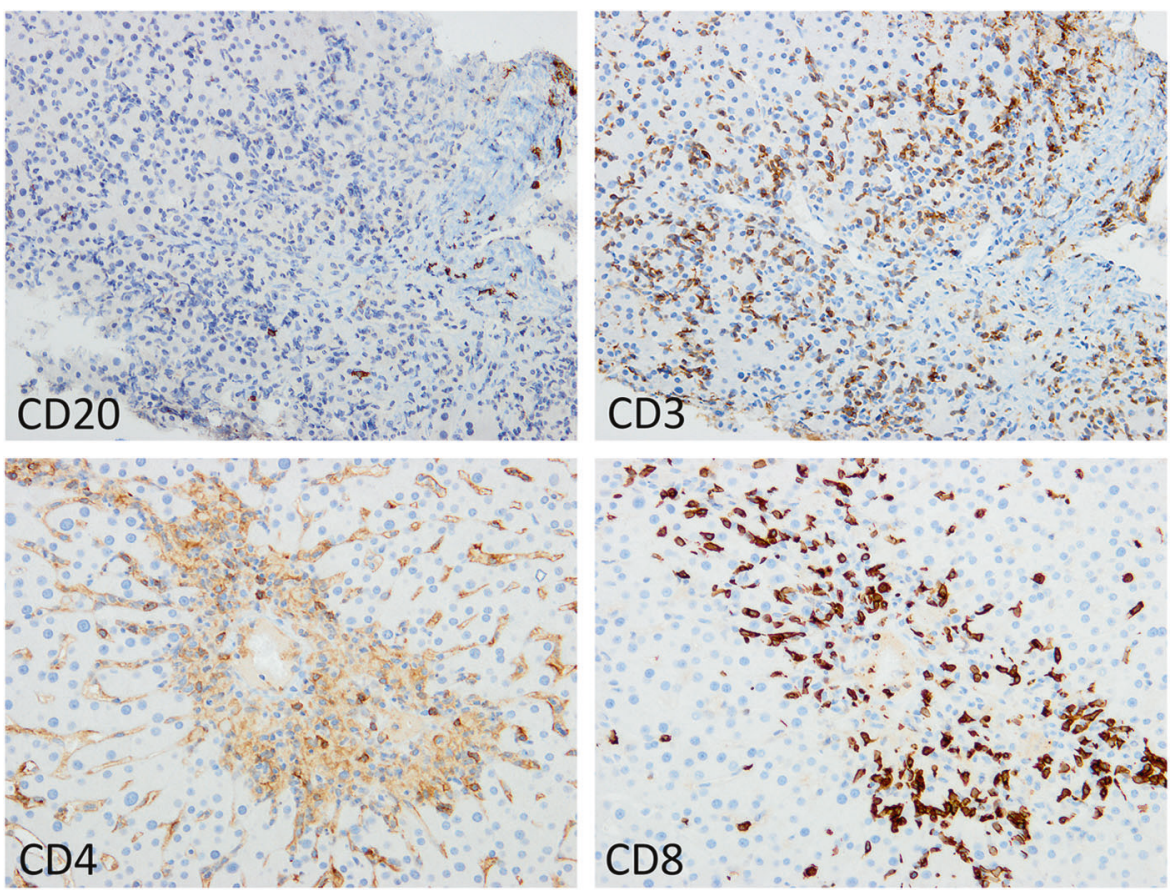

pulse therapy in one case, immunosuppression with corticosteroids appeared to be effective. These results support checkpoint inhibitor-induced liver injury being an immunemediated process.

However, differences between the two conditions were more obvious. Unlike cases of autoimmune hepatitis, all patients with checkpoint inhibitor-induced liver injury lacked antinuclear antibodies and $\operatorname{IgG}$ elevations. Histologic findings also differed in several aspects, with plasmacytosis and centrilobular confluent necrosis being uncommon in checkpoint inhibitor-induced liver damage. On immunostaining, liver injury caused by immunotherapy was 
Fig. 5 Immunohistochemical results of autoimmune hepatitis. In addition to $\mathrm{CD} 3+$ or $\mathrm{CD} 8+$ T cells, CD20+ or CD4+ lymphocytes were observed
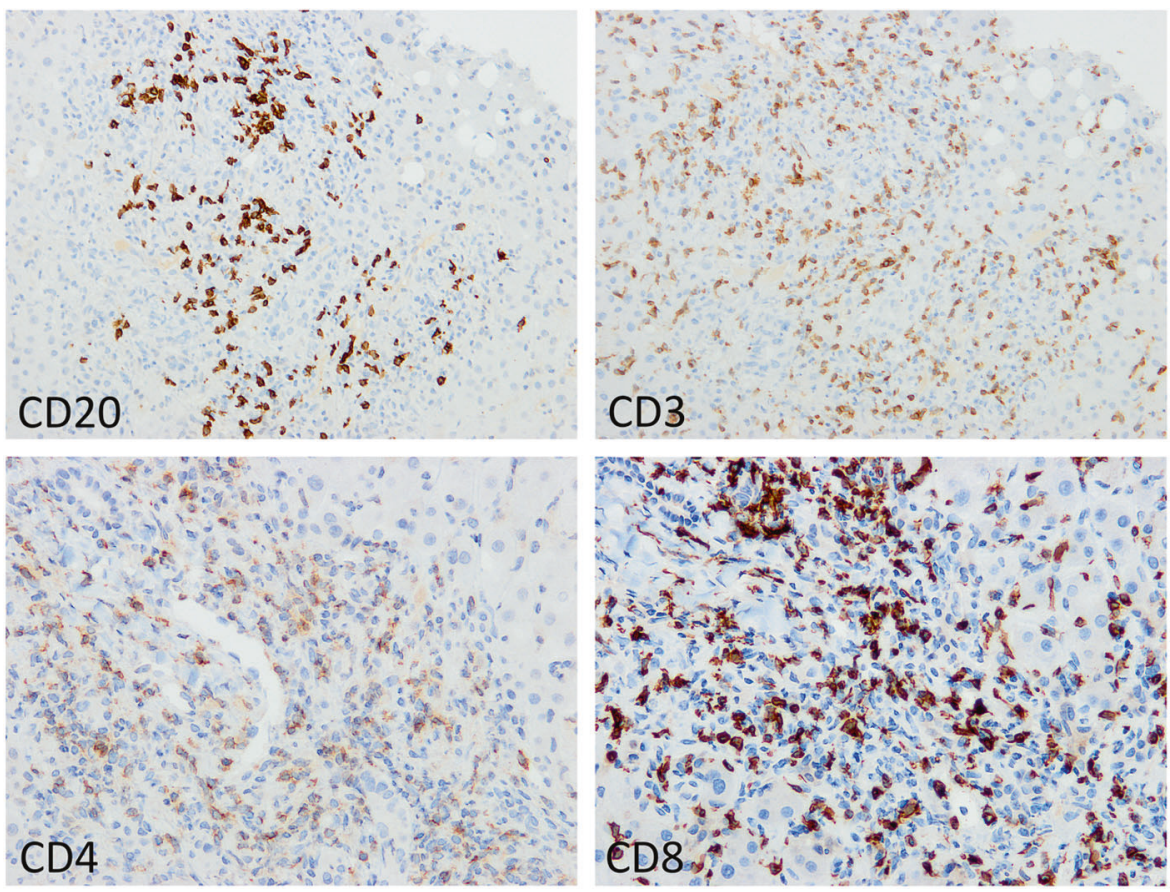

associated with markedly smaller numbers of CD20+ or CD4+ lymphocytes than autoimmune hepatitis. Differences in lymphocyte subsets may be expected because PD-1 and CTLA4 are mainly expressed by $\mathrm{CD} 8+$ cytotoxic $\mathrm{T}$ lymphocytes. Therefore, the interaction between CD4+ helper $\mathrm{T}$ cells and $\mathrm{B}$ cells may be activated less in immunotherapy-associated liver injury than in autoimmune hepatitis, which may be a reason why serum IgG levels were not elevated in this condition. This may also explain why, in contrast to autoimmune hepatitis, immunosuppression can be discontinued by tapering over 1 or 2 months in most patients with the hepatotoxicity of checkpoint inhibitors [24].

An interesting result was that confluent necrosis was rare in checkpoint inhibitor-induced liver injury, particularly nivolumab-treated cases. Zonal cell loss is common in autoimmune hepatitis and drug-induced liver injury, and typically occurs in perivenular areas. The mechanisms underlying centrilobular hepatocyte dropout have not yet been clarified; however, heterogeneity in expressed proteins and enzymatic activities among zones may explain this microscopic change. Cytochrome P450 isoforms involved in phase I drug metabolism are strongly expressed or preferentially induced in the perivenular region, while glutathione peroxidase, a phase II enzyme, displays an opposite, periportally dominated pattern [25, 26]. Therefore, proteins, enzymes, and metabolites that are more abundantly present in zone 3 hepatocytes may behave as a hepatotoxin or autoantigen triggering immune reactions in autoimmune hepatitis or drug-induced liver injury. The uncommon development of confluent necrosis in checkpoint inhibitor-induced liver injury suggests that the liver damage may represent a less zone-selective hepatocyte necrosis not requiring the strong activation of CD4+ Tlymphocytes and immunoglobulin production. Since perivenular areas are the most peripheral from a blood flow perspective, ischemia is another factor potentially contributing to zonal necrosis, and it may be a major mechanism of zonal necrosis observed, although rarely, in checkpoint inhibitor-associated liver injury.

The present study also elucidated minor histologic findings in checkpoint inhibitor-induced liver injury. Patient 1 showed features suggestive of large-duct injury including a ductular reaction and biliary-type interface activity. That case may have had additional cholangitis in the large-duct because a few recent studies suggested that nivolumab induces cholangiopathy [27, 28]. In patient $3, \sim 20$ foci of microabscesses were observed in the core of liver tissue. Microabscesses in liver biopsy samples generally indicate the possibility of infection. However, this may not have been the case in our patient because she responded well to steroid pulse therapy. Therefore, the cause of micro-abscesses remains unclear. A recent study reported fibrin ring granulomas in two patients treated with a combination of ipilimumab and nivolumab for malignant melanoma [29]. Although this was not detected in our cases, the unusual form of granuloma is another minor microscopic finding potentially observed in checkpoint inhibitor-induced liver injury.

In conclusion, checkpoint inhibitor-induced liver injury was predominantly characterized by lobular hepatitis with 
the infiltration of many CD3+ or CD8+ T-lymphocytes, consistent with an immune-mediated process. However, unlike classic autoimmune hepatitis, serological autoimmune abnormalities (e.g., antinuclear antibodies and IgG elevations), centrilobular zonal necrosis, and plasmacytosis were uncommon. Based on the small numbers of CD4+ $\mathrm{T}$ cells and $\mathrm{CD} 20+\mathrm{B}$ cells, checkpoint inhibitor-induced liver injury may represent less zone-selective hepatocyte necrosis not requiring the strong activation of helper $\mathrm{T}$ cells and immunoglobulin production.

\section{Compliance with ethical standards}

Conflict of interest The authors declare that they have no conflict of interest.

\section{References}

1. Postow MA, Callahan MK, Wolchok JD. Immune checkpoint blockade in cancer therapy. J Clin Oncol. 2015;33:1974-82.

2. Brahmer JR, Tykodi SS, Chow LQ, et al. Safety and activity of anti-PD-L1 antibody in patients with advanced cancer. N Engl J Med. 2012;366:2455-65.

3. Robert C, Long GV, Brady B, et al. Nivolumab in previously untreated melanoma without BRAF mutation. N Engl J Med. 2015;372:320-30.

4. Melosky B, Chu Q, Juergens R, et al. Pointed progress in secondline advanced non-small-cell lung cancer: The rapidly evolving field of checkpoint inhibition. J Clin Oncol. 2016;34:1676-88.

5. Borghaei H, Paz-Ares L, Horn L, et al. Nivolumab versus docetaxel in advanced nonsquamous non-small-cell lung cancer. $\mathrm{N}$ Engl J Med. 2015;373:1627-39.

6. Motzer RJ, Escudier B, McDermott DF, et al. Nivolumab versus everolimus in advanced renal-cell carcinoma. N Engl J Med. 2015;373:1803-13.

7. Motzer RJ, Rini BI, McDermott DF, et al. Nivolumab for metastatic renal cell carcinoma: Results of a randomized phase ii trial. J Clin Oncol. 2015;33:1430-7.

8. Hodi FS, O'Day SJ, McDermott DF, et al. Improved survival with ipilimumab in patients with metastatic melanoma. N Engl J Med. 2010;363:711-23.

9. Fong L, Small EJ. Anti-cytotoxic T-lymphocyte antigen-4 antibody: the first in an emerging class of immunomodulatory antibodies for cancer treatment. J Clin Oncol. 2008;26:5275-83.

10. Wolchok JD, Kluger $\mathrm{H}$, Callahan MK, et al. Nivolumab plus ipilimumab in advanced melanoma. $\mathrm{N}$ Engl $\mathrm{J}$ Med. 2013;369:122-33.

11. Melero I, Hervas-Stubbs S, Glennie M, et al. Immunostimulatory monoclonal antibodies for cancer therapy. Nat Rev Cancer. 2007;7:95-106.

12. O'Day SJ, Hamid O, Urba WJ. Targeting cytotoxic Tlymphocyte antigen-4 (CTLA-4): a novel strategy for the treatment of melanoma and other malignancies. Cancer. 2007;110:2614-27.

13. Peggs KS, Quezada SA, Chambers CA, et al. Blockade of CTLA4 on both effector and regulatory $\mathrm{T}$ cell compartments contributes to the antitumor activity of anti-CTLA- 4 antibodies. J Exp Med. 2009;206:1717-25.

14. Quezada SA, Simpson TR, Peggs KS, et al. Tumor-reactive CD4 (+) T cells develop cytotoxic activity and eradicate large established melanoma after transfer into lymphopenic hosts. J Exp Med. 2010;207:637-50.

15. Francisco LM, Salinas VH, Brown KE, et al. PD-L1 regulates the development, maintenance, and function of induced regulatory T cells. J Exp Med. 2009;206:3015-29.

16. Weber JS, Kahler KC, Hauschild A. Management of immunerelated adverse events and kinetics of response with ipilimumab. J Clin Oncol. 2012;30:2691-7.

17. Abdel-Wahab N, Shah M, Suarez-Almazor ME. Adverse events associated with immune checkpoint blockade in patients with cancer: A systematic review of case reports. PLoS ONE. 2016;11: e0160221.

18. Kim KW, Ramaiya NH, Krajewski KM, et al. Ipilimumab associated hepatitis: imaging and clinicopathologic findings. Invest New Drugs. 2013;31:1071-7.

19. Johncilla M, Misdraji J, Pratt DS, et al. Ipilimumab-associated Hepatitis: Clinicopathologic characterization in a series of 11 cases. Am J Surg Pathol. 2015;39:1075-84.

20. Kleiner DE, Berman D. Pathologic changes in ipilimumab-related hepatitis in patients with metastatic melanoma. Dig Dis Sci. 2012;57:2233-40.

21. Hennes EM, Zeniya M, Czaja AJ, et al. Simplified criteria for the diagnosis of autoimmune hepatitis. Hepatology. 2008;48:169-76.

22. Ishak K, Baptista A, Bianchi L, et al. Histological grading and staging of chronic hepatitis. J Hepatol. 1995;22:696-9.

23. Simonelli M, Di Tommaso L, Baretti M, et al. Pathological characterization of nivolumab-related liver injury in a patient with glioblastoma. Immunotherapy. 2016;8:1363-9.

24. Huffman BM, Kottschade LA, Kamath PS, et al. Hepatotoxicity after immune checkpoint inhibitor therapy in melanoma: Natural progression and management. Am J Clin Oncol. 2017. https://doi. org/10.1097/COC.0000000000000374.

25. Lindros KO. Zonation of cytochrome P450 expression, drug metabolism and toxicity in liver. Gen Pharmacol. 1997;28:191-6.

26. Oinonen T, Lindros KO. Zonation of hepatic cytochrome P-450 expression and regulation. Biochem J. 1998;329(Pt 1):17-35.

27. Gelsomino F, Vitale G, Ardizzoni A. A case of nivolumab-related cholangitis and literature review: how to look for the right tools for a correct diagnosis of this rare immune-related adverse event. Invest New Drugs. 2017. https://doi.org/10.1007/s10637-0170484-6.

28. Kawakami H, Tanizaki J, Tanaka K, et al. Imaging and clinicopathological features of nivolumab-related cholangitis in patients with non-small cell lung cancer. Invest New Drugs. 2017;35:529-36.

29. Everett J, Srivastava A, Misdraji J. Fibrin ring granulomas in checkpoint inhibitor-induced hepatitis. Am J Surg Pathol. 2017;41:134-7. 